\title{
Improving the safety and efficiency of nurse medication rounds through the introduction of an automated dispensing cabinet
}

Alan Cottney

East London NHS Foundation Trust

\begin{abstract}
Information technology (IT) systems are being utilised with increasing frequency at the prescribing and dispensing stage of the medicines-use process in UK hospitals. However, much less development has taken place with regard to the implementation of IT systems at the administration stage of medicines-use. A technology that has been implemented widely at the administration stage in North American hospitals is the automated dispensing cabinet (ADC), which has been shown to reduce nurse medication administration errors and reduce the time that nurses spend administering medication. The current project was undertaken to assess whether these benefits would be realised with the introduction of an ADC on an inpatient ward in a UK mental health hospital.
\end{abstract}

Nurses were observed administering medication before and after the implementation of an ADC on a ward at East London NHS Foundation Trust (ELFT). The findings from these observations showed that the use of the ADC led to a reduction in the medication administration error rate from $8.9 \%$ to $7.2 \%$; however, this reduction was solely accounted for by a reduction in errors of negligible clinical severity. The types of administration errors noted after implementation of the ADC remained largely unchanged from beforehand. The ADC was found to reduce the amount of time that nurses spent administering medication from $2.94 \mathrm{~min}$ per dose to $2.37 \mathrm{~min}$ per dose. It is estimated that this reduction could generate around 66 min of additional free nursing time per ward per day.

As a standalone device, the ADC was found to improve the efficiency of the medicines-use process, but had little meaningful effect on medication administration error rate at ELFT. However, it could be anticipated that additional benefit with regard to reducing medication administration errors may be demonstrated if the ADC was used in combination with other IT systems, such as electronic prescribing.

\section{Problem}

At its most basic level, the medicines-use process on inpatient hospital wards is frequently viewed as a series of chronological steps, beginning with prescribing, followed by dispensing, and then proceeding to administration to the patient (1). In the UK, information technology (IT) systems have become an increasingly common presence in the medicines-use process. A burgeoning number of hospital trusts have implemented systems such as electronic prescribing and web based decision support tools aimed at improving the safety and efficiency of medication prescribing. Similarly, IT systems, such as automated pharmacy "robots", are commonly employed at the dispensing stage of the medicines-use process. Despite the apparent embrace of technology to assist prescribing and dispensing, there has been little progress with regard to the use of IT systems at the administration stage of medicines-use in UK hospitals. As a result, the method of medication administration on the majority of hospital wards has remained largely unchanged for decades. This reluctance to adopt innovative technology to aid medication administration may mean that unsafe and inefficient systems remain in place, potentially adversely affecting the quality of patient care.

\section{Background}

Preventable errors can, and do, occur at each stage of the medicines-use process, but errors are most frequently reported to occur at the administration stage $(2,3)$. Systematic reviews of the literature have highlighted that medication administration errors may occur in around $20-25 \%$ of all opportunities $(4,5)$. Medication administration errors have the potential to harm patients severely; they can also result in the imposition of significant financial burdens on healthcare providers, due to remedial treatment costs and potential litigation expenses (6).

In addition to being error prone, the medication administration process is time consuming, with studies suggesting that up to a third of a nurse's working day will be spent on some aspect of the medication administration process (4). In mental health hospital trusts, such as East London NHS Foundation Trust (ELFT), decreased time spent administering medication could free nurses to engage patients in other clinically important therapeutic activities which could have a positive impact on patient outcomes and experience.

There is evidence to suggest that, overall, IT systems can have a generally positive impact in health settings, improving the safety, quality and efficiency of care provision $(7,8)$. With regards to the use of IT aimed specifically at the administration stage of medicinesuse, the most commonly implemented system in North American hospitals is the automated dispensing cabinet (ADC) - also known as a unit based cabinet, or an automated dispensing device. ADCs are locking cabinets that control access to medication. Nurses can 
gain access to the cabinet by using a password, or by scanning their fingerprint. A touchscreen is then used to select the required patient from a list, and then to select the medication needed for administration. When the medication is selected, the cabinet unlocks to allow access only to the location in which that particular medication is stored, and guides the nurse to that location so that they can remove the required dose. Such cabinets have now been introduced in more than $80 \%$ of US hospitals (9), and have been shown to have the potential to reduce medication errors and reduce the amount of time that nurses spend administering medication $(10,11)$.

The limitation of the current evidence with regards to the benefit of ADCs is that most of the studies were conducted in general hospitals in North America. There are significant differences in how medication is used between North America and the UK (for example, unit dose dispensing - where each dose is packaged and labelled individually for a specific patient - is common in North America, but not in the UK), and between the mental health setting and the general hospital setting. This means that the findings from the studies that have investigated the impact of ADCs are not necessarily translatable to the situation at ELFT. The objective of the current study was to assess if the benefits of ADCs with regard to reducing administration errors and reducing nurse time spent administering medication would be realised in the UK mental health hospital setting.

\section{Baseline Measurement}

A 21-bed acute adult inpatient mental health ward at ELFT was selected to take part in the project. A pharmacist, trained in the direct observation method of medication administration error detection (12), observed each of the four daily medication administration rounds that occurred on the ward during weekdays for three consecutive weeks - recording the relevant details about what they observed. At ELFT, prescriptions are handwritten on medication administration record charts, with separate charts for each patient. Any unintentional or inappropriate deviation from the prescriber's clear instructions on these charts was classed as an error, as was any obvious failure to adhere to the UK Nursing and Midwifery Council's Standards for practice of administration of medicines (13). Any administration errors that were observed were classified by type according to the headings set out in the United States' National Coordinating Council for Medication Error Reporting and Prevention (NCC MERP) Taxonomy of Medication Errors (14), which comprises the following categories: dose omission, improper dose, wrong strength/concentration, wrong drug, wrong dosage form, wrong technique, wrong route of administration, wrong rate, wrong duration, wrong time, wrong patient, monitoring error, deteriorated drug error, and other. The severity of observed errors was categorised according to the system previously reported by Haw et al (15). Any dose of medication that was prescribed to be administered, or that was administered, on the observed medication round was classed as one opportunity for error. Doses could only be classed as either in error or not in error. Total opportunities for error was used as the denominator for calculating error rate. When the pharmacists observed an error was about to reach the patient, they intervened before the medication was actually administered. For the sake of the study, such near misses were classed as administration errors. A senior pharmacist reviewed the documented errors to ensure that they did indeed represent genuine administration errors, and to ensure that they agreed with the classification of the type and severity of the error.

To obtain baseline measurements, a total of 60 medication rounds were observed -15 at each of the four daily medication round times. In total, there were 1542 observed opportunities for error; of this total number of opportunities, 138 errors were made, giving an error per opportunity rate of $8.9 \%$. The types of errors that were observed are detailed in table 1 . Forty per cent $(55 / 138)$ of the observed errors were of negligible clinical severity, and $60 \%$ $(83 / 138)$ were of minor clinical severity (table 2 provides examples of errors that were classed under each of these severities). The nurses took an average of $2.94 \mathrm{~min}$ to administer each dose of medication.

See supplementary file: ds3002.docx - "Baseline measurements"

\section{Design}

After reviewing the literature, it became apparent that the implementation of ADCs in other hospitals was associated with a reduction in medication errors and nurse time spent administering medication. Further investigation revealed that an ADC may be particularly suited to medication administration in mental health settings, such as ELFT. Nurses administering medication on a mental health ward generally remain in one location while the patients come to them to receive their dose. This is in contrast to the medication round that is most common in general hospitals which involves the nurse travelling to each patient's bedside. This may increase the utility of ADCs in the mental health setting, as the nurse does not have to move from the area in which the cabinet is placed and so can administer medication directly from the ADC.

The project was designed to assess whether any benefits could be realised from ADCs at ELFT through a small, localised roll-out. This project would then inform the decision about a wider implementation of ADCs at ELFT. If the purported benefits of ADCs reported in the literature were also realised at ELFT, the implementation of the devices would be cost-effective through equivalent savings generated from reduced nurse time spent administering medication and reduced expenditure caused by medication administration errors.

\section{Strategy}

After the baseline observation period was complete, a proprietary $A D C$ was acquired on a trial basis. The electronic cabinet was initially installed in a non-clinical area while all ward staff received comprehensive one-to-one training on how to use the device. The cabinet was then installed on the chosen ward in the treatment room from which the nurse usually administers medication. The ward that was chosen for the current project was nominated because it was felt to be representative of many of the other wards 
at ELFT in terms of patient demographics, staffing levels, and usual medicines-use processes. The ADC replaced the traditional medication storage systems on the ward, namely, a traditional locking medication trolley and two locking medication cupboards. The nurses were given 2 months to become familiar with the use of the cabinet before the post-implementation monitoring of effect took place. During this 2-month familiarisation period, a trained member of pharmacy staff was made available for the ward to contact should they have any problems pertaining to the new ADC.

\section{Results}

Two months after the ADC was implemented on the chosen ward, the direct observation process that was used to collect baseline data was repeated. Again, each of the four daily medication rounds was observed on each weekday during a 3-week observation period. The same pharmacist observers were used to collect data, and the same criteria for classification of error type and severity were used to categorise the data.

After the introduction of the ADC, a total of 60 medication rounds were observed -15 at each of the four daily medication round times. In total, there were 1895 observed opportunities for error; of this total number of opportunities, 137 errors were actually made, giving an error per opportunity rate of $7.2 \%$. The types of errors that were observed are detailed in table 3 . Twenty-six per cent $(35 / 137)$ of the observed errors were of negligible clinical severity, $72 \%$ $(100 / 138)$ were of minor clinical severity, and $1.5 \%(2 / 137)$ were of serious clinical severity (table 4 gives examples of the different error severities). After the implementation of the ADC, the nurses took an average of $2.37 \mathrm{~min}$ to administer each dose of medication.

It was found that, overall, the implementation of an ADC led to a reduction in the medication administration error rate from $8.9 \%$ to $7.2 \%$. This $1.7 \%$ reduction in error rate was not found to be statistically significant $(\mathrm{p}=0.065,95 \% \mathrm{Cl} 0 \%$ to $3.5 \%$, unpaired $\mathrm{t}$ test), thus it cannot be ruled out that the observed reduction was solely the result of chance. Also, the reduction in error rate was not found to be clinically significant as it was entirely accounted for by a reduction in errors of negligible severity (errors that would not be expected to have any effect on the patient). Errors in which an effect on the patient might be evident (minor, serious or fatal errors) were not reduced. The rate of occurrence of such non-negligible errors before ADC implementation was $5.4 \%$ (83/1542) and afterwards it remained at $5.4 \%(102 / 1895)$. There was no large variation in the types of medication administration error that were seen before or after the introduction of the ADC.

The average time taken for nurses to administer a dose of medication decreased from $2.94 \mathrm{~min}$ to $2.37 \mathrm{~min}$ after the implementation of the ADC, a saving of $0.57 \mathrm{~min}(p=0.006,95 \% \mathrm{Cl}$ 0.17 to $0.97 \mathrm{~min}$, unpaired $\mathrm{t}$ test). At the rate of medication administration observed in the current project (approximately 115 doses administered per ward per day), this would correspond to a total saving of roughly 66 min per ward per day of nursing time.

See supplementary file: ds3003.docx - "Table 3 \& 4- post implementation"

\section{Lessons and Limitations}

One of the main lessons learnt during this project was the importance of good communication when it comes to the implementation of changes to systems. Initially, nursing staff were wary of the new ADC device, as they thought it would increase their workload, and they were wary of being observed by the pharmacist as they felt like their competence was being called into question. These concerns were allayed by clearly communicating the fact that the implementation of the ADC was on a trial basis - so if it did not work, it would be removed - and by communicating the fact that pharmacists were there to assess the performance of the ADC, not the nurses. Good communication was also vital when coordinating the employees who were required to help with the implementation of the ADC, such as the IT department staff, estates and facilities staff, and ADC engineering staff.

Another lesson taken from the project was that the direct observation method is a very useful tool for the detection and quantification of medication administration errors. The method accurately and sensitively detected errors, and could be used to clearly show the post-intervention change (or lack of change) in error rate. The method could usefully be applied to other projects which aimed to reduce medication errors.

In terms of the technology itself, a lesson learnt was that ADCs could improve the efficiency of medicines administration at ELFT, but would probably have little effect on the rate of medication errors.

The main limitation of the project was that it was restricted to just one ward in one hospital, so wider generalisation of results is difficult. Another problem was that carrying out post-intervention measurement after only 2 months of the system being in place may have meant that nursing staff were still in the process of getting used to the device. If measurement had been conducted after a substantially longer period of time post-implementation, it may have revealed more data about the effect of the ADC after it had been thoroughly embedded into a clinical environment.

A limitation with regard to the implemented technology was that the $A D C$ was used alone, without other clinical electronic systems, such as electronic prescribing and patient barcode scanning. It could be anticipated that linking the ADC with additional systems such as these would generate much better improvements in medicines safety, and so this warrants future investigation.

\section{Conclusion}

It was hoped that installing an ADC would improve patient safety and decrease the amount of time that nurses spend administering medication. While it appears the ADC was a success in regard to the latter objective, the benefit of the cabinet in terms of patient safety was much less obvious. The overall medication administration error rate decreased from $8.9 \%$ to $7.2 \%$, but the nonnegligible error rate did not change. In other words, the rate of clinically significant administration errors was not reduced by implementing the ADC. It may be that ADCs improve patient safety 


\section{BMJ Quality Improvement Reports}

when linked to other information technology systems, such as electronic prescribing and patient barcode scanning, but when an $A D C$ was used as a standalone device no improvement was apparent. However, the saving of 66 min a day of nursing time generated by the $A D C$ is significant, and it remains for healthcare providers to decide whether this additional clinical time would correspond to a saving commensurate with the investment that would be required to install the cabinets in clinical areas.

\section{References}

1. Smith J. Department of Health. Building a safer NHS for patients: improving medication safety. London: Department of Health, 2003.

2. National Patient Safety Agency. National Reporting and Learning Service. Safety in doses: improving the use of medicines in the NHS. London: NPSA, 2009.

3. Soerensen AL, Lisby M, Nielsen LP, et al. The medication process in a psychiatric hospital: are errors a potential threat to patient safety? Risk Management and Healthcare Policy 2013;6:23-31

4. Keers RN, Williams SD, Cooke J, et al. Prevalence and nature of medication administration errors in health care settings: a systematic review of direct observational evidence. Ann Pharmacother 2013;47:237-56.

5. Berdot S, Gillaizeau F, Caruba T, et al. Drug administration errors in hospital inpatients: a systematic review. PLOS ONE 2013;8(6):1-11.

6. Kohn LT, Corrigan JM, Donaldson MS, eds. To err is human: building a safer health system. Washington, DC: National Academy Press, 1999.

7. Agrawal A. Medication errors: prevention using information technology systems. Br J Clin Pharmacol 2009;67:681-6.

8. Buntin MB, Burke MF, Hoaglin MC, et al. The benefits of health information technology: a review of the recent literature shows predominantly positive results. Health Affairs 2011;30:464-71.

9. Pedersen CA, Schneider PJ, Scheckelhoff DJ. ASHP national survey of pharmacy practice in hospital settings: dispensing and administration-2008. Am J Health Syst Pharm 2009;66:926-46.

10. Skibinski KA, White BA, Lin LI-K, et al. Effects of technological interventions on the safety of a medicationuse system. Am J Health Syst Pharm 2007;64:90-6.

11. Borel JM, Rascati KL. Effect of an automated, nursing unitbased drug-dispensing device on medication errors. Am J Health Syst Pharm 1995;52:1875-9.

12. Barker KN, McConnell WE. The problems of detecting medication errors in hospitals. Am J Hosp Pharm 1962;19:360-9.

13. Nursing and Midwifery Council. Standards for medicines management (2010). NMC London. http://www.nmc-uk.org/ Documents/NMC-Publications/NMC-Standards-formedicines-management.pdf (accessed 18 March 2013).

14. National Coordinating Council for Medication Error Reporting and Prevention. NCC MERP Taxonomy of Medication Errors.

http://www.nccmerp.org/pdf/taxo2001-07-31.pdf (accessed
18 March 2013).

15. Haw C, Stubbs J, Dickens G. An observational study of medication administration errors in old-age psychiatric inpatients. Int J Qual Health Care 2007;19:210-16.

\section{Declaration of interests}

Nothing to declare.

\section{Acknowledgements}

I would like to acknowledge the kind assistance of the members of staff on Conolly Ward and in the Pharmacy Department, East London NHS Foundation Trust. 